\title{
Réponse au Di-Ammonium Phosphate des Rendements en Feuilles, en Calices et en Graines des Écotypes d'oseille de Guinée (Hibiscus sabdariffa L.) au Niger
}

\author{
Kaka Kiari Boukar Kellou, \\ Inoussa Maman Maârouhi,
}

Université Abdou Moumouni de Niamey Faculté des Sciences et Techniques

Département de Biologie Laboratoire de Recherche en Gestion et

Valorisation de la Biodiversité au Sahel, Niamey, Niger

Abasse Amadou Tougiani,

Institut National de la Recherche Agronomique du Niger, Niamey, Niger

Moussa Massaoudou,

Institut National de la Recherche Agronomique du Niger, Département de

Gestion des Ressources Naturelles, Maradi, Niger

Atta Sanoussi,

Centre Régional AGRHYMET, Niamey, Niger

Bakasso Yacoubou,

Université Abdou Moumouni de Niamey Faculté des Sciences et Techniques

Département de Biologie, Niamey, Niger

Doi:10.19044/esj.2019.v15n21p470 URL:http://dx.doi.org/10.19044/esj.2019.v15n21p470

\section{Résumé:}

$\mathrm{Au}$ Sahel, l'oseille est cultivée pour ses feuilles, ses graines et ses calices qui constituent une source de revenu pour les producteurs. Les essais ont été conduits dans les stations de recherche de l'Institut National de la Recherche Agronomique (INRAN) de Tara/Gaya et Tarna/Maradi au Niger au cours des saisons de pluies 2015 et 2016 pour étudier l'effet de fertlisation minérale sur les écotypes d'oseille. Le dispositif expérimental était un split plot en blocs randomisés à quatre répétitions. Deux facteurs ont été étudiés : les écotypes d'oseille avec six niveaux (E1, E4, E5, E6, E7 et E8) et la fertilisation sous forme de Di-Ammonium Phosphate (DAP) apportée à 3 différentes doses $(0 \mathrm{~kg} / \mathrm{ha}, 25 \mathrm{~kg} / \mathrm{ha}$ et $50 \mathrm{~kg} / \mathrm{ha})$. A la récolte, les rendements en feuilles, calices et graines ont été évalués dans un carré de rendement de $10 \mathrm{~m}^{2}$. Cette étude a montré que l'apport de fertilisation minérale (DAP) a permis d'augmenter les rendements en feuilles de $50 \%$, en calices de $45 \%$ et en graines $30 \%$ sur le site de Tara/Gaya au cours de deux années 
expérimentations. Cependant, sur Tarna/Maradi, cette augmentation a été de $20 \%$ comparée aux témoins. La dose optimale de DAP qui améliore les rendements en feuilles et en calices au niveau des deux sites est de $50 \mathrm{~kg} / \mathrm{ha}$. Pour les rendements en graines, elle est de $25 \mathrm{~kg} / \mathrm{ha}$. Les rendements enregistrés sur le site de Tara/Gaya étaient supérieurs à ceux de Tarna/Maradi au cours des deux années d'expérimentation. Enfin, les rendements ont été plus élevés en 2015 au niveau des deux sites.

Mots clés : Oseille, écotypes, rendements, DAP, calices, feuilles, graines, Niger

\title{
Di-Ammonium Phosphate Response to Leaf, Calyx Ans Seed Yields of Roselle (Hibiscus sabdariffa L.) Ecotypes in Niger
}

\section{Kaka Kiari Boukar Kellou, Inoussa Maman Maârouhi,}

Université Abdou Moumouni de Niamey Faculté des Sciences et Techniques

Département de Biologie Laboratoire de Recherche en Gestion et

Valorisation de la Biodiversité au Sahel, Niamey, Niger

Abasse Amadou Tougiani,

Institut National de la Recherche Agronomique du Niger, Niamey, Niger

Moussa Massaoudou,

Institut National de la Recherche Agronomique du Niger, Département de

Gestion des Ressources Naturelles, Maradi, Niger

Atta Sanoussi,

Centre Régional AGRHYMET, Niamey, Niger

\section{Bakasso Yacoubou,}

Université Abdou Moumouni de Niamey Faculté des Sciences et Techniques

Département de Biologie, Niamey, Niger

\begin{abstract}
In the Sahel, sorrel is grown for its leaves, seeds and calyxes which provide a source of income for producers. The trials were conducted in the research stations of the National Institute for Agricultural Research (INRAN) of Tara/Gaya and Tarna/Maradi in Niger during the 2015 and 2016 rainy seasons to study the effect of mineral fertilization on the sorrel ecotypes. The
\end{abstract}


experimental device was a split plot in randomized blocks with four repetitions. Two factors were studied: the sorrel ecotypes with six levels (E1, E4, E5, E6, E7 and E8) and the fertilization in the form of Di-Ammonium Phosphate (DAP) provided at 3 different doses $(0 \mathrm{~kg} / \mathrm{ha}, 25 \mathrm{~kg} / \mathrm{ha}$ and $50 \mathrm{~kg} / \mathrm{ha})$. At harvest, yields of leaves, calices and seeds were evaluated in a yield square of $10 \mathrm{~m}^{2}$. This study showed that the mineral fertilization supplement (DAP) allowed increasing leaf yields of $50 \%$, $45 \%$ calyx and $30 \%$ seeds at the Tara/Gaya site during two years of experimentation. . However, on Tarna/Maradi, this increase was $20 \%$ compared to the witnesses. The optimal dose of DAP that improves leaf and calyx yields at both sites is $50 \mathrm{~kg} / \mathrm{ha}$. For seed yields, it is $25 \mathrm{~kg} / \mathrm{ha}$. The yields recorded at the Tara/Gaya site were higher than at Tarna/Maradi during the two years of experimentation. Lastly, yields were higher in 2015 at both sites.

Keywords: Sorrel, ecotypes, yields, DAP, calyx, leaves, seeds, Niger

\section{Introduction}

L'oseille de Guinée (Hibiscus sabdariffa L.) est principalement cultivée dans les pays du Sahel comme le Niger. Les feuilles et les calices de la plante sont utilisés sous forme de légume ou de décoction (Bakasso, 2010; McClintock et al., 2011; Adamou, 2013). Au Niger, les jeunes feuilles sont également utilisées comme ingrédients dans les sauces et servent de complément nutritif aux céréales comme le riz, le sorgho et le mil. Les graines sont transformées en soumbala et représentent une importante source de revenu pour les producteurs (Bengaly et al., 2006; Barazi, 2009; Bakasso, 2010). La plante est également utilisée pour soigner de nombreuses maladies comme la toux, les maux de dents et les troubles de l'hypertension (Bérhaut, 1979; Hassane, 2005).

Du point de vue nutritionnelle, les feuilles sont riches en $\mathrm{Ca}, \mathrm{K}, \mathrm{Mg}, \mathrm{N}$ et P (Atta et al., 2010a). Les calices sont plus riches en $\mathrm{Ca}, \mathrm{K}, \mathrm{Mn}, \mathrm{Na}$ et $\mathrm{Fe}$ que les graines (Atta et al., 2013). Les graines d'oseille contiennent environ $60 \%$ de protéines, $20 \%$ de matières grasses, $15 \%$ d'huile, $5 \%$ de cendres et de glucides (Halimatu et $a l ., 2007$; Mera et $a l .$, 2009). L'oseille de Guinée est également cultivée pour sa fibre dans certaines zones de production (Mera et al., 2009; Maunde, 2010; Mohammed et al., 2013).

La culture de l'oseille de Guinée se fait généralement au Niger en association avec d'autres cultures, mais le plus souvent en bordure des champs (Bakasso, 2010). Les cultures pures sont très rares et pratiquées par les femmes sur de petites parcelles, autour du village à côté des grands champs de céréales (Bakasso, 2010; McClintock et al., 2011). Malgré les avantages qu'offre cette culture, au Niger le rendement est généralement faible (Seyni, 2005) en raison parfois du potentiel génétique limité des variétés utilisées et surtout d'un 
manque d'apport de fertilisants par les producteurs (Hussein et al., 2006). Pourtant les travaux sur la fertilisation de l'oseille de Guinée ont montré les effets positifs d'un apport d'engrais minéral sur la production d'oseille (Egharevba et Law-Ogbomo, 2007; Majeed et Ali, 2011; Atta et al., 2010; Mehdi, 2012; Said et al., 2015). Aussi, la mise à la disposition des producteurs des doses de fertilisation minérale en fonction des zones agro-climatiques et des objectifs de production est indispensable pour un programme d'intensification et de valorisation de la culture d'oseille au Niger.

L'objectif de cette étude est de déterminer la dose optimale de DiAmmonium Phosphate (DAP) pour la production de feuilles, calices et graines d'oseille de Guinée dans deux zones agro-climatiques du Niger.

\section{Matériel et Méthodes}

\section{Présentation des sites expérimentaux}

Les essais ont été conduits au cours des campagnes agricoles 2015 et 2016 au niveau des stations expérimentales de l'Institut National de la Recherche Agronomique du Niger (INRAN) à Tara/Gaya (Figure 1) et à Tarna/Maradi (Figure 2). La station de Tara/Gaya est située à $310 \mathrm{~km}$ au sudest de Niamey $\left(11^{\circ} 53^{\prime} \mathrm{N}\right.$ et $\left.3^{\circ} 19^{\prime} \mathrm{E}\right)$ dans la zone nord soudanienne. Celle de Tarna/Maradi $\left(13^{\circ} 27^{\prime} \mathrm{N}\right.$ et $\left.7^{\circ} 06^{\prime} \mathrm{E}\right)$ est à $657 \mathrm{~km}$ à l'Est de Niamey, dans la zone agro-climatique sahélienne. Les sols des deux sites expérimentaux sont sableux, acides et pauvres en matière organique (Tableau 1). Le sol de Tara/Gaya est plus riche en azote que celui de Tarna/Maradi mais plus pauvre en Phosphore-Bray 1.

Les quantités de pluies enregistrées au cours des deux années d'expérimentation ont été plus élevées à Tara/Gaya $(689,62 \mathrm{~mm}$ en 2015 et $782,29 \mathrm{~mm}$ en 2016) qu'à Tarna/Maradi (respectivement, 450,57 $\mathrm{mm}$ et 496,6 $\mathrm{mm}$ ). L'année 2016 a été plus pluvieuse que 2015 dans les deux sites (Figure $1)$. 


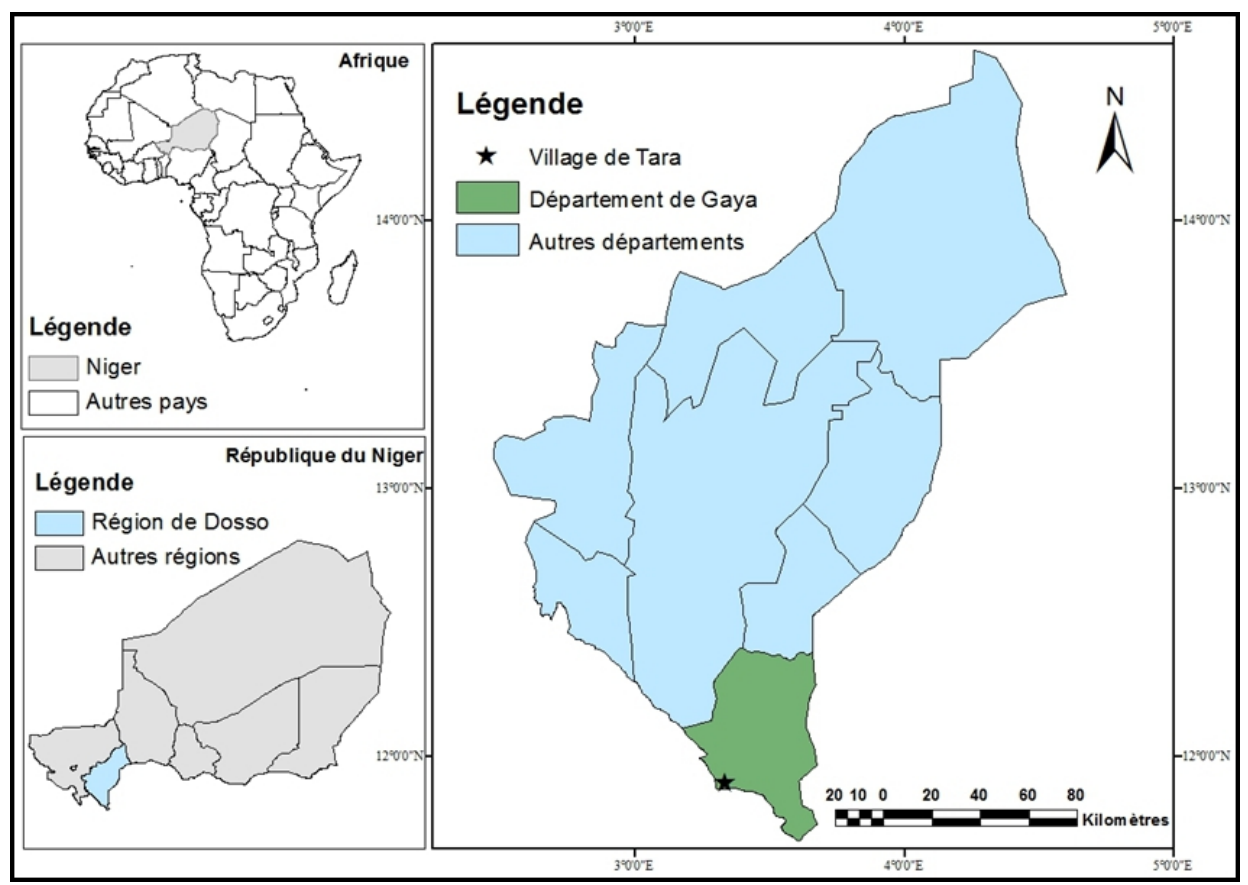

Figure 1: Localisation de Tara/Gaya

Tableau 1 : Caractéristiques physico-chimiques de Tara/Gaya et de Tarna/Maradi à $0-15 \mathrm{~cm}$ et $15-30 \mathrm{~cm}$.

\begin{tabular}{lcccccc}
\hline & \multicolumn{2}{c}{ Tara/Gaya } & & \multicolumn{2}{c}{ Tarna/Maradi } \\
\cline { 2 - 3 } \cline { 5 - 6 } Composition du sol & $\mathbf{0 - 1 5} \mathbf{~ c m}$ & $\mathbf{1 5 - 3 0} \mathbf{~ c m}$ & & $\mathbf{0 - 1 5} \mathbf{~ c m}$ & $\mathbf{1 5 - 3 0} \mathbf{~ c m}$ \\
\hline Argile (\%) & 6,92 & 10,20 & & 0,40 & 2,10 \\
Limon Fin (\%) & 2,30 & 1,80 & & 0,70 & 0,50 \\
Limon Gros (\%) & 4,83 & 5,20 & & 1,10 & 0,70 \\
Sable Fin (\%) & 49,87 & 48,20 & & 42,30 & 42,10 \\
Sable Gros (\%) & 36,07 & 34,60 & & 55,50 & 54,60 \\
\hline Propriétés chimiques & 3,92 & 3,9 & & 5 & 4,40 \\
pH/KCl & 4,95 & 5 & & 5,9 & 5,70 \\
pH/H & 2,74 & 4,30 & & 6,40 & 7,20 \\
Phosphore-Bray1 (mg/kg) & 0,19 & 0,20 & & 0,10 & 0,10 \\
Carbone Organique (\%) & 163,03 & 142,90 & & 96,50 & 71,10 \\
Azote total (mg/kg) & & & & &
\end{tabular}

\section{Matériel végétal et dispositif expérimental}

Le matériel végétal de cette étude a été collecté au Niger dans le cadre des activités du Laboratoire de Gestion et Valorisation de la Biodiversité au Sahel de la Faculté des Sciences et Techniques de l'Université Abdou Moumouni de Niamey (Bakasso et al., 2008). Ce matériel est composé de six (6) écotypes d'oseille de Guinée provenant de quatre régions du Niger (Tableau 2). Ces écotypes se différencient par le type botanique, la couleur des 
calices et des feuilles, la forme des feuilles et le type de production (calices, feuilles, graines).

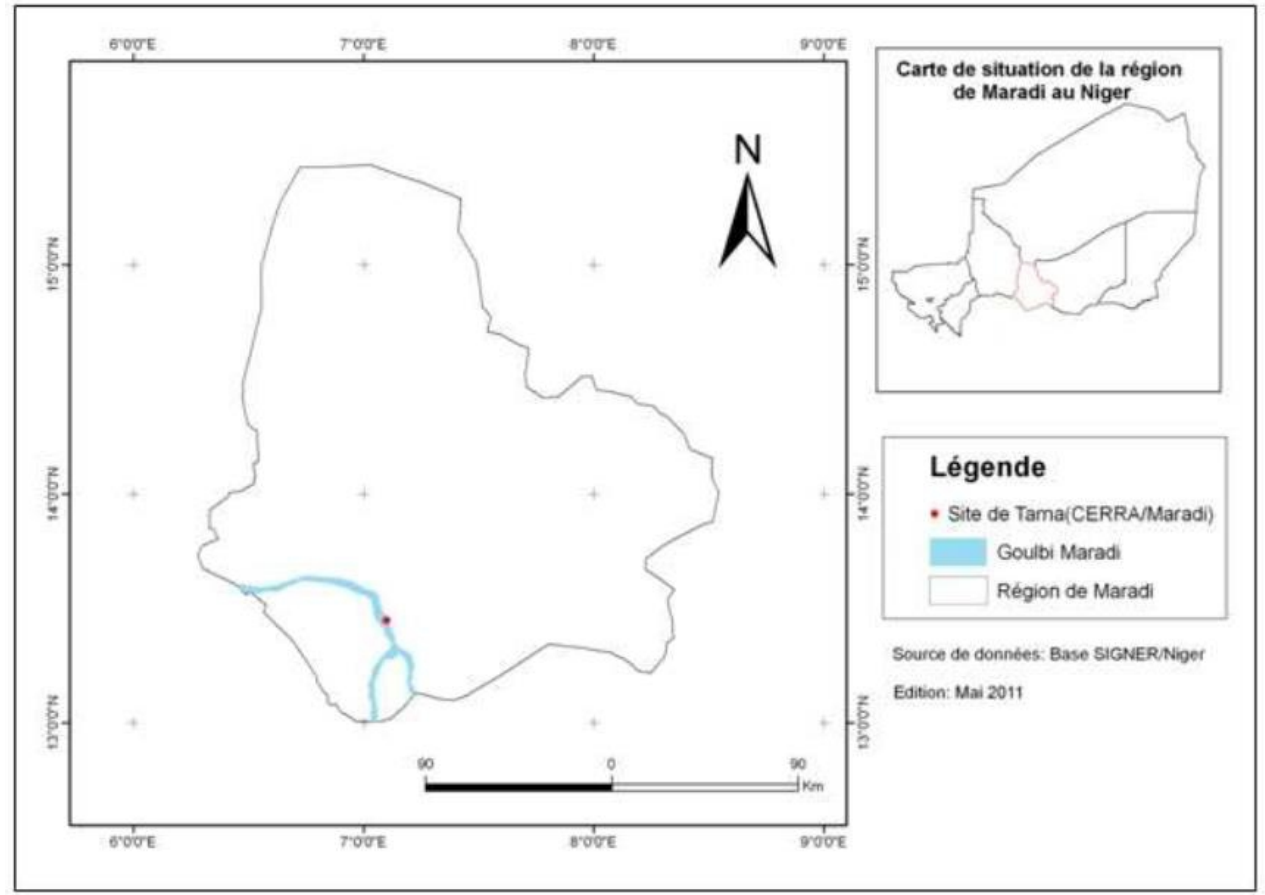

Figure 2: Localisation de Tarna/Maradi

Tableau 2 : Région d'origine, types botaniques et caractéristiques des différents écotypes étudiés.

\begin{tabular}{|c|c|c|c|c|c|c|}
\hline Ecotypes & $\begin{array}{l}\text { Région } \\
\text { d'origine }\end{array}$ & $\begin{array}{c}\text { Type } \\
\text { botanique }\end{array}$ & $\begin{array}{c}\text { Couleur } \\
\text { des calices }\end{array}$ & $\begin{array}{c}\text { Formes des } \\
\text { feuilles }\end{array}$ & $\begin{array}{l}\text { Couleur } \\
\text { des tiges }\end{array}$ & $\begin{array}{c}\text { Type de } \\
\text { production }\end{array}$ \\
\hline E1 & Tillabéry & Yakua poilu & Blanche rayée & Trilobées longues & Rouge & Graines et feuilles \\
\hline E4 & Zinder & Waré & Blanche & Trilobées larges & Rouge & Calices blancs \\
\hline E5 & Maradi & Waré & Rouge claire & Trilobées larges & Rouge & Calices rouges \\
\hline E6 & Maradi & Yakua & Blanche & Simple ovales & Blanche & Feuilles et graines \\
\hline E7 & Maradi & Waré & Verte & Trilobées larges & Verte & Calices verts \\
\hline E8 & Dosso & Waré & Noire & $\begin{array}{l}\text { Pentalobée } \\
\text { longues }\end{array}$ & Noire & Calices noirs \\
\hline
\end{tabular}

Source Bakasso et al., 2008.

Le dispositif expérimental était un split plot en blocs randomisés à quatre (4) répétitions. Deux facteurs ont été étudiés: les écotypes d'oseille avec les six niveaux en parcelles principales et la fertilisation sous forme de DAP apportée à 3 différentes doses $(0 \mathrm{~kg} / \mathrm{ha}, 25 \mathrm{~kg} / \mathrm{ha}$ et $50 \mathrm{~kg} / \mathrm{ha})$ disposés en sousparcelles. Chaque parcelle expérimentale a une superficie de $20 \mathrm{~m}^{2}(6 \mathrm{~m}$ x 5 $\mathrm{m})$. Les répétitions successives ont été séparées par un espacement de $2 \mathrm{~m}$.

Les parcelles ont été labourées à l'aide d'un tracteur, suivi d'un hersage et d'un enfouissement des résidus de récolte de la culture précédente. Le semis 
d'oseille a été réalisé dans les deux sites à raison de 5 graines par poquet, le 14 juillet en 2015 et le 20 juillet en 2016. Il a été démarié un plant par poquet. L'apport de DAP contenant $18 \%$ de $\mathrm{N}$ et $46 \%$ de $\mathrm{P}$ a été fait au stade végétatif après une pluie utile.

\section{Paramètres mesurés}

La maturité était intervenue à Tarna/Maradi et à Tara/Gaya en moyenne respectivement au $105^{\text {ème }}$ et $120^{\text {éme }}$ jours après le semis. Les paramètres suivants ont été mesurés à la récolte par plante dans un carré de rendement de $10 \mathrm{~m}^{2}(4 \mathrm{~m} \times 2,5 \mathrm{~m})$ par parcelle : le poids sec de graines, le poids sec de calices et le poids sec de feuilles.

L'indice de récolte en graines (IRG) a été calculé par la formule de (Huehn Manfred, 1993) :

$$
\text { IRG }(\%)=\frac{\text { MSG }}{\text { MST }} \times 100(1) \quad \text { MST }=\text { MSG }+ \text { MSF }+ \text { MSC (2) }
$$

Où : MSG est la Matière Sèche des Graines ; MSF est la Matière Sèche des Feuilles ;

MSC est la Matière Sèche des Calices ; MST est la Matière Sèche Totale.

L'indice de récolte en feuilles (IRF) et en calices (IRC) a également été calculé.

\section{Analyse statistique des données}

L'analyse de variance (ANOVA) à deux facteurs a été faite à l'aide du logiciel GenStat version 12.1 pour évaluer les effets du DAP, des écotypes et leur interaction. La séparation des moyennes pour les différents paramètres mesurés a été réalisée par le test de Student Newman Keuls au seuil de signification de $5 \%$.

\section{Résultats}

\section{Rendement en feuilles}

Les résultats de l'analyse de variance indiquent des différences significatives $(\mathrm{P}<0,001)$ de rendement en feuilles entre les trois doses de DAP au niveau des deux sites. Le meilleur rendement en feuilles a été enregistré à la dose de 50kg/ha au cours des deux années à Tara/Gaya et en 2015 à Tarna/Maradi et le plus faible rendement par le témoin (Tableau 3). En effet, l'apport de $50 \mathrm{~kg} / \mathrm{ha}$ de DAP a permis d'augmenter le rendement en feuilles d'environ 50\% à Tara/Gaya aussi bien en 2015 qu'en 2016. Cette augmentation est cependant plus faible à Tarna/Maradi (environ $20 \%$ en 2015). Le rendement en feuilles n'était pas significativement différent entre les doses de $25 \mathrm{~kg}$ de DAP/ha et $50 \mathrm{~kg} / \mathrm{ha}$ en 2016 à Tara/Gaya et en 2015 à Tarna/Maradi. Cependant, aucune différence significative de rendement en 
feuilles n'a été enregistrée entre les trois doses de DAP à Tarna/Maradi en 2016. L'interaction entre les doses de DAP et les écotypes n'a pas été significative au niveau des deux sites et pour les deux années d'expérimentation (Tableau 3).

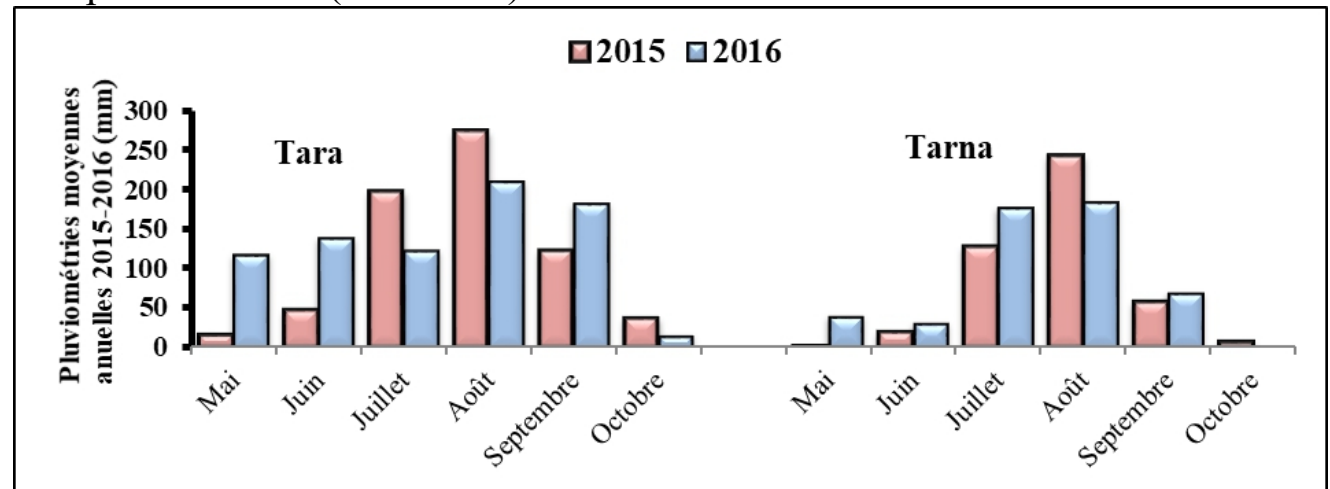

Figure 3 : Distribution de la pluviométrie mensuelle à Tara/Gaya et à Tarna/Maradi durant les deux années d'expérimentations (2015 et 2016).

Tableau 3 : Effet de doses de DAP et des écotypes d'oseille sur le rendement $(\mathrm{kg} / \mathrm{ha})$ en feuilles en 2015 et 2016 à Tara/Gaya et Tarna/Maradi.

\begin{tabular}{|c|c|c|c|c|}
\hline \multirow[b]{2}{*}{ Ecotypes } & \multicolumn{2}{|c|}{ Tara/Gaya } & \multicolumn{2}{|c|}{ Tarna/Maradi } \\
\hline & 2015 & 2016 & 2015 & 2016 \\
\hline E1 & $1080 \mathrm{~b}$ & $1093,3 a$ & 833,2 & $435,7 \mathrm{ab}$ \\
\hline E4 & $971 b$ & $859,2 b$ & 1001,4 & $369,9 \mathrm{c}$ \\
\hline E5 & $1423 a$ & $856,0 \mathrm{~b}$ & 894,0 & $394,0 b c$ \\
\hline E6 & $790 \mathrm{c}$ & $918,8 b$ & 908,6 & $445,2 \mathrm{ab}$ \\
\hline E7 & $1400 \mathrm{a}$ & $947,7 b$ & 979,5 & $370,7 \mathrm{c}$ \\
\hline E8 & $1268 \mathrm{a}$ & $792,3 b$ & 960,8 & $457,4 \mathrm{a}$ \\
\hline Moyenne \pm ET & $1155,3 \pm 251,92$ & $911,2 \pm 104,34$ & $929,58 \pm 62,6$ & $412,15 \pm 38,79$ \\
\hline \multicolumn{5}{|l|}{ Doses de DAP } \\
\hline $0 \mathrm{Kg} / \mathrm{ha}$ & $935 c$ & $689,2 b$ & $853,3 b$ & 397,0 \\
\hline $25 \mathrm{Kg} / \mathrm{ha}$ & $1095 b$ & $998,9 a$ & $938,5 \mathrm{ab}$ & 427,9 \\
\hline $50 \mathrm{Kg} / \mathrm{ha}$ & $1435 a$ & $1045,6 a$ & $997,1 \mathrm{a}$ & 411,6 \\
\hline Moyenne \pm ET & $1155 \pm 225,34$ & $911,2 \pm 193,70$ & $929,6 \pm 72,30$ & $412,1 \pm 1,45$ \\
\hline Ecotypes & $* * *$ & $* *$ & ns & $* * *$ \\
\hline Doses & $* * *$ & $* * *$ & $*$ & ns \\
\hline Ecotypes $\times$ Doses & ns & ns & ns & ns \\
\hline
\end{tabular}

Des différences significatives du rendement en feuilles existent également entre les deux années au niveau des deux sites. En effet, ce rendement est plus élevé en 2015 qu'en 2016, particulièrement à Tarna/Maradi où il a diminué de moitié (respectivement $929 \mathrm{~kg} / \mathrm{ha}$ et $412 \mathrm{~kg} / \mathrm{ha}$ ).

Des différences significatives $(\mathrm{P}<0,001)$ de rendement en feuilles existent également entre les écotypes aussi bien en 2015 qu'en en 2016 à 
Tara/Gaya (Tableau 3). En 2015, les écotypes E5, E7 et E8 ont donné les meilleurs rendements en feuilles, respectivement $1423 \mathrm{~kg} / \mathrm{ha}, 1400 \mathrm{~kg} / \mathrm{ha}$ et $1268 \mathrm{~kg} / \mathrm{ha}$. L'écotype E6 a le plus faible rendement $(790 \mathrm{~kg} / \mathrm{ha})$. Cependant, en 2016, l'écotype E1 a enregistré le meilleur rendement en feuilles (1093,3 $\mathrm{kg} / \mathrm{ha}$ ) comparativement à tous les autres écotypes qui ont un rendement similaire qui se situe entre 790 et $950 \mathrm{~kg} / \mathrm{ha}$. A Tarna/Maradi, aucune différence significative de rendement en feuilles n'a été enregistrée entre les écotypes en 2015 (Tableau 3). Cependant en 2016, le meilleur rendement a été obtenu pour E8 (457kg/ha) et le plus faible pour E4 et E7 (370 kg/ha). Les autres écotypes ont des rendements intermédiaires.

\section{Rendement en calices}

L'apport de doses de DAP se traduit par une augmentation significative $(\mathrm{P}<0,05)$ du rendement en calices des écotypes au cours des deux années et au niveau des deux sites (Tableau 4). C'est ainsi que les apports de $25 \mathrm{~kg} / \mathrm{ha}$ et $50 \mathrm{~kg} / \mathrm{ha}$ se traduisent par des rendements en calices similaires mais supérieurs au témoin. Les augmentations de rendements se situent autour de $20 \%$ par rapport au témoin à Tarna/Maradi pour les deux années et à Tara/Gaya en 2015. Cette augmentation est encore plus importante à Tara/Gaya en 2016 (45\% du témoin).

A Tara/Gaya, le rendement en calices enregistré en 2016 (705 kg/ha) a connu une augmentation de $80 \%$ par rapport à celui de 2015 (395 kg/ha). Par contre à Tarna/Maradi, il est passé de 538 kg/ha en 2015 à $317 \mathrm{~kg} / \mathrm{ha}$ en 2016, soit une diminution de $40 \%$ (Tableau 4).

L'analyse de variance montre aussi une différence significative des rendements en calices en $2015(\mathrm{P}<0,05)$ et en $2016(\mathrm{P}<0,001)$ entre les écotypes à Tara/Gaya (Tableau 4). En 2015, le meilleur rendement a été obtenu par l'écotype E8 (530 kg/ha) et le plus faible (307 kg/ha) par les écotypes E1, E6 et E7. Cependant en 2016, E1 a enregistré le meilleur rendement en calices $(910 \mathrm{~kg})$. Les autres écotypes ont un rendement similaire, compris entre 600 et $700 \mathrm{~kg} / \mathrm{ha}$.

En 2015 à Tarna/Maradi, le meilleur rendement en calices a été obtenu au niveau des écotypes E4, E5 et E7 (autour de $650 \mathrm{~kg} / \mathrm{ha}$ ), et le plus faible rendement $(260 \mathrm{~kg} / \mathrm{ha})$ par l'écotype E1. Cependant aucune différence significative n'a été enregistrée entre les écotypes en 2016. Aucune interaction significative n'a également été enregistrée entre les écotypes et les doses de DAP au niveau des deux sites et au cours des deux années d'expérimentation (Tableau 4). 
Tableau 4: Effet de doses de DAP et des écotypes d'oseille sur les rendements (en $\mathrm{kg} / \mathrm{ha}$ ) en calices en 2015 et 2016 à Tara/Gaya et Tarna/Maradi.

\begin{tabular}{|c|c|c|c|c|}
\hline \multirow[b]{2}{*}{ Ecotypes } & \multicolumn{2}{|c|}{ Tara/Gaya } & \multicolumn{2}{|c|}{ Tarna/Maradi } \\
\hline & 2015 & 2016 & 2015 & 2016 \\
\hline E1 & $351,7 \mathrm{bc}$ & $910,2 a$ & $260,8 \mathrm{c}$ & 313,8 \\
\hline E4 & $407,5 b$ & $729,6 b$ & $660,5 a$ & 319,8 \\
\hline E5 & $412,3 b$ & $655,2 b$ & $632,0 \mathrm{a}$ & 317,3 \\
\hline E6 & $358,4 b c$ & $709,2 b$ & $516,1 \mathrm{~b}$ & 327,9 \\
\hline E7 & $307,5 \mathrm{c}$ & $628,8 b$ & $670,7 \mathrm{a}$ & 309,8 \\
\hline E8 & $530,5 a$ & $599,6 b$ & $489,5 b$ & 314,3 \\
\hline Moyenne \pm ET & $394,6 \pm 77,05$ & $705,4 \pm 111,46$ & $538,26 \pm 155,7$ & $317,15 \pm 6,26$ \\
\hline Doses de DAP & & & & \\
\hline $0 \mathrm{Kg} / \mathrm{ha}$ & $355,1 b$ & $561,9 b$ & $460,5 b$ & $292,1 b$ \\
\hline $25 \mathrm{Kg} / \mathrm{ha}$ & $405,3 \mathrm{ab}$ & $736,5 a$ & $581,1 \mathrm{a}$ & $315,0 \mathrm{ab}$ \\
\hline $50 \mathrm{Kg} / \mathrm{ha}$ & $423,5 \mathrm{a}$ & $817,9 a$ & $573,1 \mathrm{a}$ & $344,3 a$ \\
\hline Moyenne \pm ET & $394,6 \pm 35,42$ & $705,4 \pm 130,79$ & $538,2 \pm 67,43$ & $317,1 \pm 26,16$ \\
\hline Ecotypes & $* * *$ & $* * *$ & $* * *$ & ns \\
\hline Doses & $*$ & $* * *$ & $* *$ & $* *$ \\
\hline Ecotypes $\times$ Doses & ns & ns & ns & ns \\
\hline
\end{tabular}

Les chiffres portant la (ou les) même(s) lettre(s) dans la même colonne ne sont pas significativement différents au seuil de $\mathrm{p}<0,05 ; \mathrm{ns}=$ non significatif; $*=$ significatif $; * *=$ hautement significatif; $* * *=$ très hautement significatif, ET= Ecart-Type.

\section{Rendement en graines}

A Tara/Gaya en 2015, l'apport de DAP n'a pas eu un effet significatif $(\mathrm{P}>0,05)$ sur les rendements en graines des écotypes qui est de l'ordre de 607 $\mathrm{kg} / \mathrm{ha}$ (Tableau 5). Cependant, en 2016, une différence très hautement significative $(\mathrm{P}<0,001)$ a été enregistrée entre le témoin et les autres doses de DAP qui ont un rendement en graines similaires. L'apport de DAP a permis d'augmenter le rendement d'environ $40 \%$ par rapport au témoin. A Tarna/Maradi, l'apport de DAP se traduit également par une augmentation significative $(\mathrm{P}<0,01)$ du rendement en graines au cours des deux années. Cependant, le rendement n'était pas significativement différent entre les doses de $25 \mathrm{~kg} / \mathrm{ha}$ et $50 \mathrm{~kg} / \mathrm{ha}$ de DAP. Par rapport au témoin, l'augmentation du rendement en graines est plus importante en 2015 (26\%) comparée à celle de 2016 (15\%). L'interaction entre les doses de DAP et les écotypes n'a pas été significative au niveau des deux sites et pour les deux années d'expérimentation.

Les résultats de l'analyse de variance indiquent des différences significatives entre les écotypes aussi bien à Tara/Gaya qu'à Tarna/Maradi au cours des deux années d'expérimentation (Tableau 5). A Tara/Gaya en 2015, le meilleur rendement en graines a été obtenu pour l'écotype E1 (1082 kg/ha) et le plus faible rendement par les écotypes E4, E5, E7 et E8. Cependant en 2016, les écotypes E5, E7 et E8 ont obtenu un rendement en graines similaire 
(autour de $600 \mathrm{~kg} / \mathrm{ha}$ ) et plus élevé que les autres écotypes qui se situent autour de $430 \mathrm{~kg} / \mathrm{ha}$.

A Tarna/Maradi, le meilleur rendement en graines a également été enregistré en 2015 par l'écotype E1 (877 kg/ha).Le plus faible rendement a été enregistré pour E8 (444 kg/ha). Les autres écotypes ont un rendement en graines similaire. En 2016, le meilleur rendement en graines a été obtenu pour l'écotype E7 (362 kg/ha) et le plus faible pour E1 (202 kg/ha) (Tableau 5).

Tableau 5 : Effet de doses de DAP et des écotypes d'oseille sur les rendements (en $\mathrm{kg} / \mathrm{ha}$ ) en graines en 2015 et 2016 à Tara/Gaya et Tarna/Maradi.

\begin{tabular}{|c|c|c|c|c|}
\hline \multirow[b]{2}{*}{ Ecotypes } & \multicolumn{2}{|c|}{ Tara/Gaya } & \multicolumn{2}{|c|}{ Tarna/Maradi } \\
\hline & 2015 & 2016 & 2015 & 2016 \\
\hline E1 & $1082,5 \mathrm{a}$ & $432,3 b$ & $877,4 a$ & $201,6 \mathrm{c}$ \\
\hline E4 & $534,8 \mathrm{c}$ & $465,2 b$ & $712,1 b$ & $312,9 b$ \\
\hline E5 & $372,2 \mathrm{c}$ & $633,8 \mathrm{a}$ & $686,3 b$ & $290,4 b$ \\
\hline E6 & $777,1 b$ & $412,9 b$ & $626,3 b$ & $269,0 \mathrm{~b}$ \\
\hline E7 & $398,0 \mathrm{c}$ & $595,6 \mathrm{a}$ & $664,0 \mathrm{~b}$ & $361,6 a$ \\
\hline E8 & $476,4 \mathrm{c}$ & $597,7 \mathrm{a}$ & $444,5 c$ & $283,3 b$ \\
\hline Moyenne \pm ET & $606,8 \pm 274,26$ & $522,9 \pm 96,74$ & $668,43 \pm 139,88$ & $286,46 \pm 52,68$ \\
\hline \multicolumn{5}{|l|}{ Doses de DAP } \\
\hline $0 \mathrm{Kg} / \mathrm{ha}$ & 547,7 & $420,0 b$ & $567,6 \mathrm{~b}$ & $259,2 b$ \\
\hline $25 \mathrm{Kg} / \mathrm{ha}$ & 660,0 & $555,1 \mathrm{a}$ & $737,8 \mathrm{a}$ & $295,1 \mathrm{a}$ \\
\hline $50 \mathrm{Kg} / \mathrm{ha}$ & 612,7 & $593,6 \mathrm{a}$ & $697,9 a$ & $305,1 \mathrm{a}$ \\
\hline Moyenne \pm ET & $606,8 \pm 56,38$ & $522,9 \pm 91,16$ & $667,7 \pm 89,01$ & $286,4 \pm 24,13$ \\
\hline Ecotypes & $* * *$ & $* * *$ & $* * *$ & $* * *$ \\
\hline Doses & ns & $* * *$ & $* *$ & $* *$ \\
\hline Ecotypes $\times$ Doses & ns & ns & ns & ns \\
\hline
\end{tabular}

Les chiffres portant la (ou les) même(s) lettre(s) dans la même colonne ne sont pas significativement différents au seuil de $\mathrm{p}<0,05$. ns = non significatif; $*=$ significatif ; $* *=$ hautement significatif; $* * *=$ très hautement significatif, ET= Ecart-Type.

\section{Indice de récolte en feuilles}

Les résultats de l'analyse de variance montrent également des différences significatives de l'indice de récolte des feuilles entre les trois doses de DAP (Tableau 6). Cependant, l'effet du DAP varie suivant l'année et le site expérimental. En 2015 au niveau de deux sites, le meilleur indice de récolte a été enregistré pour le traitement témoin et pour la dose de $50 \mathrm{~kg}$ de DAP/ha qui ont un indice de récolte en feuilles similaires. Cependant cet indice était plus élevé à Tarna/Maradi (24,00\%) qu'à Tara/Gaya (19,70\%). En 2016 à Tara/Gaya, le meilleur indice de récolte en feuilles a été enregistré à la dose de $25 \mathrm{~kg}$ de DAP/ha et le plus faible pour le témoin. Par contre à Tarna/Maradi, le témoin et la dose de $25 \mathrm{~kg}$ de $\mathrm{DAP} / \mathrm{ha}$ ont un indice de récolte similaire et supérieur à celui de la dose de $50 \mathrm{~kg} / \mathrm{ha}$ (Tableau 6). 
Les indices de récolte en feuilles diffèrent significativement entre les écotypes au cours des deux années d'expérimentation et au niveau des deux sites (Tableau 6).

A Tara/Gaya en 2015, le meilleur indice de récolte des feuilles a été enregistré pour les écotypes E5, E7 et E8 (autour de 22,5\%) et le plus faible $(13,85 \%)$ pour l'écotype E6. Cependant en 2016, E6 avait le meilleur indice de récolte $(24,94 \%)$ alors que les écotypes E4 et E8 avaient le plus faible $(22,70 \%)$.

A Tarna/Maradi, le meilleur indice de récolte en feuilles $(26,80 \%)$ a été obtenu pour l'écotype E8 aussi bien en 2015 qu'en 2016. Le plus faible indice de récolte a été enregistré en 2015 pour les écotypes E5 et E6, et en 2016 pour les écotypes E4, E5 et E7. Les autres écotypes avaient des indices de récolte en feuilles intermédiaires (Tableau 6).

Tableau 6 : Effet de doses de DAP et des écotypes d'oseille sur l'indice de récolte en feuilles (en \%)en 2015 et 2016 à Tara/Gaya et Tarna/Maradi.

\begin{tabular}{|c|c|c|c|c|}
\hline \multirow[b]{2}{*}{ Ecotypes } & \multicolumn{2}{|c|}{ Tara/Gaya } & \multicolumn{2}{|c|}{ Tarna/Maradi } \\
\hline & 2015 & 2016 & 2015 & 2016 \\
\hline E1 & $17,11 \mathrm{~b}$ & $24,26 a b$ & $22,87 \mathrm{ab}$ & $24,92 \mathrm{ab}$ \\
\hline E4 & $16,17 b$ & $22,60 \mathrm{~b}$ & $25,29 \mathrm{ab}$ & $21,85 \mathrm{bcd}$ \\
\hline E5 & $22,70 \mathrm{a}$ & $23,58 \mathrm{ab}$ & $20,73 b$ & $22,97 \mathrm{bcd}$ \\
\hline E6 & $13,85 \mathrm{c}$ & $24,94 \mathrm{a}$ & $21,83 b$ & 24,89abc \\
\hline E7 & $23,45 \mathrm{a}$ & $24,28 \mathrm{ab}$ & $25,43 \mathrm{ab}$ & $22,00 \mathrm{bcd}$ \\
\hline E8 & $21,34 \mathrm{a}$ & $22,84 b$ & $26,87 \mathrm{a}$ & $26,73 a$ \\
\hline Moyenne \pm ET & $19,10 \pm 3,92$ & $23,75 \pm 0,90$ & $23,83 \pm 2,38$ & $23,89 \pm 1,93$ \\
\hline \multicolumn{5}{|l|}{ Doses de DAP } \\
\hline $0 \mathrm{Kg} / \mathrm{ha}$ & $19,58 \mathrm{a}$ & $23,04 \mathrm{~b}$ & $25,54 \mathrm{a}$ & $25,44 a$ \\
\hline $25 \mathrm{Kg} / \mathrm{ha}$ & $17,93 \mathrm{~b}$ & $24,40 \mathrm{a}$ & $22,12 b$ & $23,97 \mathrm{a}$ \\
\hline $50 \mathrm{Kg} / \mathrm{ha}$ & $19,79 a$ & $23,81 \mathrm{ab}$ & $23,85 \mathrm{ab}$ & $22,27 b$ \\
\hline Moyenne $\pm E T$ & $19,10 \pm 1,01$ & $23,75 \pm 0,68$ & $23,83 \pm 1,71$ & $23,89 \pm 1,58$ \\
\hline Ecotypes & $* * *$ & $* *$ & $* *$ & $* * *$ \\
\hline Doses & * & $*$ & $*$ & $* * *$ \\
\hline Ecotypes $\times$ Doses & ns & ns & ns & ns \\
\hline
\end{tabular}

Les chiffres portant la (ou les) même(s) lettre(s) dans la même colonne ne sont pas significativement différents au seuil de $\mathrm{p}<0,05 . \mathrm{ns}=$ non significatif; $*=$ significatif $; * *=$ hautement significatif; $* * *=$ très hautement significatif, ET= Ecart-Type.

\section{Indice de récolte en calices}

A Tara/Gaya en 2015, la dose de $25 \mathrm{~kg}$ de DAP/ha (14,50\%) a obtenue le meilleur indice de récolte en calices et le plus faible $(12,98 \%)$ à la dose de $50 \mathrm{~kg} / \mathrm{ha}$ (Tableau 7). En 2016, le témoin a le plus faible indice de récolte en calices $(16,91 \%)$. Les doses de 25 et $50 \mathrm{~kg}$ de DAP/ha ont un indice de récolte similaire qui se situe autour de $20,00 \%$. 
A Tarna/Maradi, aucune différence significative d'indices de récolte en calices n'a été enregistrée entre les trois doses de DAP aussi bien en 2015 qu'en 2016. Cependant l'indice moyen de récolte en calices enregistré en 2015 $(19,55 \%)$ est supérieur à celui de 2016 (16,53\%) (Tableau 7).

Tableau 7 : Effet de doses de DAP et des écotypes d'oseille sur l'indice de récolte en calices (en \%)en 2015 et 2016 à Tara/Gaya et Tarna/Maradi.

\begin{tabular}{|c|c|c|c|c|}
\hline \multirow[b]{2}{*}{ Ecotypes } & \multicolumn{2}{|c|}{ Tara/Gaya } & \multicolumn{2}{|c|}{ Tarna/Maradi } \\
\hline & 2015 & 2016 & 2015 & 2016 \\
\hline E1 & $10,34 d$ & $13,73 b$ & $10,66 c$ & $11,52 \mathrm{e}$ \\
\hline E4 & $15,80 \mathrm{~b}$ & $15,55 b$ & $22,25 \mathrm{ab}$ & $18,37 \mathrm{a}$ \\
\hline E5 & $14,85 b$ & $22,82 \mathrm{a}$ & $21,91 \mathrm{ab}$ & $16,66 \mathrm{c}$ \\
\hline E6 & $12,84 \mathrm{c}$ & $15,55 b$ & $19,33 b$ & $14,89 \mathrm{~d}$ \\
\hline E7 & $11,41 \mathrm{~cd}$ & $22,16 a$ & $22,84 a$ & $21,14 a$ \\
\hline E8 & $18,54 \mathrm{a}$ & $24,07 \mathrm{a}$ & $20,31 \mathrm{ab}$ & $16,60 \mathrm{c}$ \\
\hline Moyenne $\pm \mathbf{E T}$ & $13,96 \pm 3,03$ & $19,09 \pm 4,51$ & $19,55 \pm 4,54$ & $16,53 \pm 3,23$ \\
\hline \multicolumn{5}{|l|}{ Doses de DAP } \\
\hline $0 \mathrm{Kg} / \mathrm{ha}$ & $14,63 \mathrm{a}$ & $16,91 b$ & 19,00 & 16,60 \\
\hline $25 \mathrm{Kg} / \mathrm{ha}$ & $14,29 a$ & $20,42 \mathrm{a}$ & 20,05 & 16,50 \\
\hline $50 \mathrm{Kg} / \mathrm{ha}$ & $12,98 b$ & $19,75 \mathrm{a}$ & 19,59 & 16,48 \\
\hline Moyenne \pm ET & $13,96 \pm 0,87$ & $19,09 \pm 1,86$ & $19,55 \pm 0,52$ & $16,53 \pm 0,64$ \\
\hline Ecotypes & $* * *$ & $* * *$ & $* * *$ & $* * *$ \\
\hline Doses & $*$ & $* * *$ & ns & ns \\
\hline Ecotypes $\times$ Doses & ns & ns & ns & ns \\
\hline
\end{tabular}

Les chiffres portant la (ou les) même(s) lettre(s) dans la même colonne ne sont pas significativement différents au seuil de $\mathrm{p}<0,05 . \mathrm{ns}=$ non significatif; $*=$ significatif $; * *=$ hautement significatif; $* * *=$ très hautement significatif, ET= Ecart-Type.

\section{Indice de récolte en graines}

A Tara/Gaya en 2015, les résultats de l'analyse de variance indiquent des différences significatives d'indices de récolte en graines entre les doses de DAP (Tableau 8). Les doses de $25 \mathrm{~kg}$ et de $0 \mathrm{~kg} / \mathrm{ha}$ de DAP ont eu un indice similaire qui se situe autour de $11,00 \%$. A la dose de $50 \mathrm{~kg}$ de DAP/ha, l'indice de récolte en graines n'est que de 8,58\%. Cependant en 2016, aucune différence significative n'a été enregistrée entre les doses de DAP. Mais l'indice moyen de récolte $(24,96 \%)$ est supérieur à celui de 2015 (10,33\%). Par ailleurs, à Tarna/Maradi, aucune différence significative d'indices de récolte en graines n'a été obtenue entre les doses de DAP aussi bien en 2015 qu'en 2016. L'indice moyen de récolte en graines est similaire pour ces deux années (autour de 17,00\%) (Tableau 8). 
Tableau 8 : Effet de doses de DAP et des écotypes d'oseille sur l'indice de récolte en graines (en \%)en 2015 et 2016 à Tara/Gaya et Tarna/Maradi.

\begin{tabular}{|c|c|c|c|c|}
\hline \multirow[b]{2}{*}{ Ecotypes } & \multicolumn{2}{|c|}{ Tara/Gaya } & \multicolumn{2}{|c|}{ Tarna/Maradi } \\
\hline & 2015 & 2016 & 2015 & 2016 \\
\hline E1 & $17,25 \mathrm{a}$ & $26,20 b$ & $23,26 a$ & $23,25 a$ \\
\hline E4 & $9,27 \mathrm{c}$ & $29,10 \mathrm{a}$ & $17,81 \mathrm{~b}$ & $20,21 b$ \\
\hline E5 & $5,98 d$ & $20,73 c$ & $15,94 b$ & $15,20 \mathrm{c}$ \\
\hline E6 & $14,05 b$ & $25,30 \mathrm{~b}$ & $15,01 \mathrm{~b}$ & $15,81 \mathrm{c}$ \\
\hline E7 & $6,85 \mathrm{~cd}$ & $24,24 b$ & $16,55 b$ & $17,47 \mathrm{~b}$ \\
\hline E8 & $8,56 \mathrm{~cd}$ & $24,21 b$ & $12,14 \mathrm{c}$ & $14,51 \mathrm{c}$ \\
\hline Moyenne \pm ET & $10,33 \pm 4,40$ & $24,96 \pm 2,74$ & $16,79 \pm 3,70$ & $17,74 \pm 3,38$ \\
\hline Doses de DAP & & & & \\
\hline $0 \mathrm{Kg} / \mathrm{ha}$ & $11,53 \mathrm{a}$ & 25,52 & 16,81 & 17,57 \\
\hline $25 \mathrm{Kg} / \mathrm{ha}$ & $10,93 \mathrm{a}$ & 24,33 & 17,10 & 17,84 \\
\hline $50 \mathrm{Kg} / \mathrm{ha}$ & $8,58 b$ & 25,05 & 16,45 & 17,81 \\
\hline Moyenne \pm ET & $10,33 \pm 1,55$ & $24,96 \pm 0,60$ & $16,79 \pm 0,32$ & $17,74 \pm 0,36$ \\
\hline Ecotypes & $* * *$ & $* * *$ & $* * *$ & $* * *$ \\
\hline Doses & $* * *$ & ns & ns & ns \\
\hline Ecotypes $\times$ Doses & ns & ns & ns & ns \\
\hline
\end{tabular}

Les chiffres portant la (ou les) même(s) lettre(s) dans la même colonne ne sont pas significativement différents au seuil de $\mathrm{p}<0,05$. ns = non significatif; $*=$ significatif ; $* *=$ hautement significatif; $* * *=$ très hautement significatif, ET= Ecart-Type.

\section{Discussion}

Les résultats ont montré l'existence d'une variation du rendement en feuilles, en calices et en graines des écotypes en fonction des sites et pour un même site selon les années. Les rendements moyens enregistrés à Tara/Gaya sont supérieurs à ceux de Tarna/Maradi de $20 \%$ en 2015 et $55 \%$ en 2016 pour les feuilles, de $55 \%$ pour les calices et $45 \%$ pour les graines en 2016. Cette variabilité des rendements peut être liée aux différences des sols et des conditions climatiques des deux sites. Le site de Tara/Gaya étant plus argileux et limoneux que celui de Tarna/Maradi (Tableau 1). En effet, (Bakasso, 2010) a montré que la nature du sol et le climat constituent le principal facteur de la variabilité des performances des écotypes d'oseille au Niger. Situé en zone nord soudanienne, la station de Tara/Gaya a également bénéficié au cours des deux années d'une pluviométrie de $35 \%$ supérieure à celle de Tarna/Maradi en zone sahélienne. L'importance de l'eau pour la croissance et le rendement de l'oseille de Guinée a été rapportée au Burkina Faso par (Hien, 2012). Cependant, (El Naim et Ahmed, 2010) ont également indiqué que la quantité et la distribution de la pluviométrie constituent le principal facteur limitant de la production d'oseille de cette culture au Soudan.

En effet, cette espèce a besoin de 450 à $500 \mathrm{~mm}$ de précipitations bien réparties sur 90 à 120 jours pendant sa phase végétative (McClintock et al., 
2011). Ceci laisse croire que l'oseille de Guinée préfère un sol riche en azote, argileux et bien drainé (site de Tara), mais peut aussi tolérer un sol sableux (site Tarna). C'est ainsi que (Mehdi, 2012) a rapporté que l'oseille se développe bien sur les sols fertiles, mais peut aussi tolérer les sols sableux et limoneux modérément fertiles.

L'apport de DAP a permis d'augmenter les rendements en feuilles de $50 \%$, en calices de $45 \%$ et en graines $30 \%$ du témoin à Tara/Gaya au cours de deux années expérimentations. Cependant, cette augmentation a été plus faible à Tarna/Maradi (de l'ordre de 20\%). Ces résultats sont conformes aux travaux de (Said et al., 2015; Mehdi, 2012; Haruna et al., 2011; Atta et al., 2010) qui ont montré que l'apport de la fertilisation minérale a un effet significatif sur la production d'oseille. Par ailleurs, (Majeed et Ali, 2011; Mera et al., 2009; Akanbi et al., 2009; Badran et Safwat, 2004) ont été également montré que l'engrais minéral augmente considérablement la production en feuilles, en calices et en graines de l'oseille.

Le meilleur rendement obtenu dans les parcelles fertilisées (DAP), peut s'expliquer par la disponibilité des éléments nutritifs ( $\mathrm{N}$ et $\mathrm{P}$ ) libérés par les engrais apportés, qui ont amélioré la fertilité du sol de ces deux sites caractérisés par des carences en phosphore et en azote.

L'azote est un élément essentiel à la croissance des végétaux (FAO, 2005). Par ailleurs, (FAO, 2005) a également indiqué que l'azote minéral absorbé par la plante sert à la synthèse des acides aminés. Il participe, entre autres, à la construction des tissus végétaux et entre dans la formule de nombreux constituants de structure (protides), de fonction (enzyme et coenzymes) et de réserves (de nombreux grains). Le phosphore est nécessaire à de nombreuses réactions biochimiques dans lesquelles $\mathrm{P}$ sert de substrats ou de produits tels que l'ATP et fait partie intégrante des phospholipides, des phosphoprotéines et des phosphosucres (Imrani, 2014; Ghasemi et al., 2015). Il joue un rôle physiologique sur la multiplication cellulaire dans les méristèmes (ADN, ARN), la respiration cellulaire, le transfert de l'énergie (ATP, ADP) et la photosynthèse en synergie avec l'azote et de nombreux autres éléments minéraux.

Cependant, à une certaine dose le phosphore et l'azote peuvent devenir toxiques pour les plantes. Ainsi (Atta et $a l ., 2010$ ) ont constaté sur la culture de l'oseille de Guinée, que l'application de $100 \mathrm{~kg}$ N/ha avait occasionné la baisse du rendement en graines, mais sans effet sur le rendement en calices.

Les résultats ont également montré que les écotypes qui ont obtenus les meilleurs rendements en graines, ont également les rendements élevés en feuilles, mais avec des faibles rendements en calice. Réciproquement, ceux qui ont enregistrés les meilleurs rendements en calices produisent très peu des graines. La différence observée entre les écotypes pour tous les rendements, montre la difficulté de disposer des écotypes performants pour la production à 
la fois en feuilles, en calices et en graines. Des résultats similaires ont été observés par (Adamou, 2013).

L'étude a montré que les meilleurs rendements ont été obtenus en 2015 dans les deux sites. La différence des rendements observée peut être attribuée au retard accumulé pendant le semis en 2016. En effet, la date de semis a une influence directe sur le cycle de végétation et le rendement d'oseille (Jan et Stuart, 2000; Hien, 2012; Khattak et al., 2016). C'est ainsi que (Gholam et Moosavi, 2012) ont enregistré une perte en rendement atteignant 0,07 t/ha/jour suite à un retard de semis par rapport à la date optimale.

Les indices de récolte en graines et en calices obtenus dans cette étude sont plus élevés que ceux rapportés par (Atta et al., 2010). L'effet significatif de l'apport du DAP sur les indices de récolte a été surtout observé à Tara/Gaya qui a bénéficié des meilleures conditions pluviométriques.

\section{Conclusion}

Cette étude a montré que l'apport de DAP a permis d'augmenter, par rapport au témoin, les rendements en feuilles de $50 \%$, en calices de $45 \%$ et en graines $30 \%$ à Tara/Gaya au cours de deux années expérimentations. Cependant, cette augmentation a été beaucoup plus faible à Tarna/Maradi (respectivement 20\%, 20\% et de 18\%). Les rendements en feuilles, en calices et en graines étaient plus élevés à Tara/Gaya qu'à Tarna/Maradi au cours des deux saisons expérimentales.

La dose optimale de fertilisation minérale qui permet d'améliorer les rendements en feuilles et en calices est de 50kg DAP/ha à Tara/Gaya et à Tarna/Maradi. Cependant, elle est de $25 \mathrm{~kg}$ DAP/ha pour le rendement en graines dans les deux sites.

Enfin, il serait intéressant de poursuivre cette étude dans toutes les zones agro-climatiques du Niger afin de déterminer les différentes doses de fertilisation minérale en fonction des objectifs de production (graines, calices ou feuilles).

\section{Remerciement}

Les auteurs remercient le Programme de Productivité Agricole en Afrique de l'Ouest (PPAAO) pour le financement du Projet Oseille.

\section{References:}

1. Adamou, B.I. (2013). Evaluation de la diversité agro-morphologique des ecotypes locaux de l'oseille de guinée (Hibiscus sabdariffa L.) au Niger. Mémoire présenté à l'université Dan Dicko Dan Koulodo de Maradi, pour l'obtention de diplôme de Master en Biodiversité et Gestion de l'Environnement soudanien sahélo-saharien, 77p. 
2. Akanbi, W.B. Olaniyan, A.B. Togun, A.O. Ilupeju, A.E.O. and Olaniran, O.A. (2009). The effect of organic and inorganic fertilizer on growth, calyx yield and quality of Hibiscus sabdariffa L. AmericanEurasian Journal of Sustainable Agriculture, 3, 652-657.

3. Atta, S. Diallo A. B. Sarr B. Bakasso Y. Issaka L. Saâdou M. and Glew R. H. (2010). Roselle (Hibiscus sabdariffa L.) Yield and yield components in response to nitrogen fertilization in Niger. Indian $\mathrm{J}$. Agric. Res., 44 (2): pp 96-103.

4. Atta, S. Diallo A. B. Sarr B., Bakasso Y. Saâdou M. and Glew R. H. (2010a). Variation in macro-elements and protein contents of Roselle (Hibiscus sabdariffa L.) from Niger. African Journal of Food, Agriculture, Nutrition and Development 10: 2707-2718.

5. Atta, S. Sarr B. Diallo A. B. Bakasso Y. Issaka L. and Saâdou M. (2013). Nutrients composition of calyces and seeds of three Roselle (Hibiscus sabdariffa L.) ecotypes from Niger. African Journal of Biotechnology .Vol. 12(26), pp. 4174-4178.

6. Badran, F. S. and Safwat, M. S. (2004). Response of fennel plants to organic manure and bio-fertilizers in replacement of chemical fertilization in Egypt. Journal of Agricultural Research, 82(2):247256.

7. Bakasso, Y, Saâdou M, Zongo J. D and F. Baidou-Forson, (2008). Analysis of agromorphological variability of Hibiscus sabdariffa L. in Niger. Ann. Sci Agron. Benin ; 11: 21-33.

8. Bakasso Y. (2010). Ressources génétiques des roselles (Hibiscus sabdariffa L.) du Niger: Evaluations agro-morphologique et génétique. Thèse présenté a la faculté des sciences à l'Université Abdou Moumouni de Niamey pour obtenir le grade de doctorat èssciences Mention : Science Naturelles (Génétique). p102.

9. Barazi, A. A. (2009). Effet d'un apport de fertilisation minérale sur la sévérité de Macrophomina phaseolina de l'oseille (Hibiscus sabdariffa L). Mémoire présenté pour l'obtention de diplôme de fin d'étude au Centre Régional AGRHYMET, Niamey. 48ps

10. Bengaly, M. Béré A. \& Traoré A. (2006). The chemical composition of bi-kalga, a traditional fermented roselle (Hibiscus sabdariffa) seeds condiment. Electronic Journal of Food and Plants Chemistry 1 (1): 7 11.

11. Egharevba, R.K.A. Law-Ogbomo K.E. (2007). Comparative effects of two nitrogen sources on the growth and yield of roselle (Hibiscus sabdariffa) in the rainforest region: a case study of Benin-city, Edo State, Nigeria. Journal of Agronomy 6 (1): 142-146.

12. El Naim, A. M. and Ahmed, S. E. (2010). Effect of weeding frequencies on growth and yield of two roselle (Hibiscus sabdariffa L) 
varieties under rain fed. Australian Journal of Basic and Applied Sciences, 4(9): 4250 - 4255.

13. FAO, (2005). Notions de nutrition des plantes et de fertilisation des sols. Manuel de formation, Projet Intrants, Niger, 24p.

14. Ghasemi, M. Khadijeh A. Shiva G. Morteza S. Fatemeh Z. (2015). Effect of application of Nitrogen and Potassium fertilizers on some vegetative and reproductive traits in Roselle (Hibiscus sabdariffa). International Journal of Agronomy and Agricultural Research (IJAAR) ISSN: 2223-7054 (Print) 2225-3610 (Online) http://www.innspub.net Vol. 7, No. 2, p. 75-79.

15. Gholam, S. \& Moosavi R. (2012). The effect of sowing date and plant density, on yield and yield components of roselle. Journal/Medicinal Plant Research, 6(9): 1627-1632.

16. Halimatu, S. M. Amin N. Mohd-Esa N. Nawalya A.G., and Siti Muskinah M. (2007). Protein quality of roselle (Hibiscus sabdariffa L.) seeds. ASEAN food Journal, 14:131-140.

17. Haruna, I.M. Maunde, S.M. and Yahuza, S. (2011). Growth and calyx yield of roselle (Hibiscus sabdariffa L.) as affected by poultry manure and nitrogen fertilizer rates in southern Guinea savanna of Nigeria, Canadian Journal of pure applied \&Sciences. Vol.5, No 1, pp, 13451348.

18. Hassane, S. H., 2005. Etude de la variabilité du rendement de dix écotypes d'oseille (Hibiscus sabdariffa). Mémoire présenté à la faculté d'Agronomie de l'Université Abdou Moumouni de Niamey pour l'obtention du diplôme de fin d'études, 49p.

19. Hien, N.A. (2012). Etude comparée de deux systèmes de culture (pluvial et irrigue) de la roselle (Hibiscus sabdariffa L.) dans la province du IOBA: évaluation du potentiel de production en calices. Mémoire présenté pour l'obtention de diplôme d'ingénieur du développement rural à l'Institut Du Développement Rural (Université Polytechnique De Bobo-Dioulasso), 62p.

20. Huen, M. (1993). Harvest index versus grain/straw-ratio. Theoritical comments and experimental results on the comparison of variation. Euphytica 68(1-2), 27-32.

21. Hussein, R.M. and Ibrahim, M.M. (2006). Variability, habitability and genetic advance in some genotypes of Roselle (Hibiscus sabdariffa L.). World J. Agric. Sci. 2: 340-345.

22. Imrani, N. Ouazzani Chahdi A. Chliyeh M., Touati J. Ouazzani Touhami A. Benkirane R. et Douira A. (2014). Effet de la fertilisation par différents niveaux de NPK sur le développement des maladies foliaires du riz. Journal of Animal \& Plant Sciences, 2014. Vol.23, Issue 1: 3601-3625. 
23. Khattak, A.M., M. Sajid, H.Z. Sarwar, A. Rab, M. Ahmad and M.A. Khan, (2016). Effect of sowing time and plant density on the growth and production of roselle (Hibiscus sabdariffa). Int. J. Agric. Biol., 00: 000-000

24. Majeed, K. A. and Ali, S. A. (2011). Effect of Foliar Application of NPK 20-10-10 on Some Growth Characters of Two Cultivars of Roselle (Hibiscus sabdariffa L.). American Journal of Plant Physiology, 6: 220-227.

25. Maunde, S. M. (2010). Yield components of roselle (Hibiscus sabdariffa L.) as Influenced by Nitrogen, Phosphorus and Farm yard manure. Unplublished B.Sc. Thesis, Department of Agronomy, Faculty of Agriculture, Ahmadu Bello University; Zaria. 32pp.

26. McClintock, N.C. \& El Tahir, I.M. (2011). (Hibiscus sabdariffa L). [Internet] Fiche de PROTA4U. Brink, M. \& Achigan-Dako, E.G. (Editeurs). PROTA (Plant Resources of Tropical Africa / Ressources végétales de l'Afrique tropicale), Wageningen, Pays Bas. <http://www.prota4u.org/search.asp>. Visité le 5 juillet 2015.

27. Mehdi, D. (2012). Effect of mineral and organic fertilizers on the growth and calyx yield of roselle (Hibiscus sabdariffa L.) African Journal of Biotechnology 11 (48):10899-10902.

28. Mera, U. M. Singh, B. R. Magaji, M. D. Singh, A. Musa, M. and Kilgori, M. J. S. (2009). Response of Roselle (Hibiscus sabdariffa L.) to Farmyard Manure and Nitrogen-fertilizer in the semi-arid savanna of Nigeria. Nigerian Journal of Basic and Applied Science, 17(2): 246251.

29. Mohammed, R., Emeghara, U. U., Momodu, G. G., Omonomo, P. Awotide, O. G., Sulaiman, R. and Hamma, I. L. (2013). Yield and yield components of roselle (Hibiscus sabdariffa L.) as affected by inorganic fertilizer application levels and pruning periods. Proceedings of 32nd Horticultural Society of Nigeria Organized by University of Agriculture Abeokuta 19th - 23rd October, 2014.

30. Sa'id, A. Rabo, B.S. Mustapha, A.B, Simon, S.Y. and Hamma I. L. (2015). Influence of NPK fertilizer on the performance of roselle (Hibiscus sabdariffa L.) in Samaru, Zaria. Nigerian Journal of Agriculture, Food and Environment.11 (3): 61-64

31. Seyni, H.H. (2005). Etude de la variabilité du rendement de dix écotypes d'oseille (Hibiscus sabdariffa). Mémoire présenté à la faculté d'agronomie de l'Université Abdou Moumouni de Niamey pour l'obtention de diplôme d'Ingénieur des Techniques Agricoles. 47p. 